\title{
La didáctica de la literatura: hacia la consolidación del campo'
}

Felipe Munita"

\footnotetext{
I- Este artículo se inscribe en el proyecto de investigación El efecto profesor en la formación de lectores: Un estudio exploratorio sobre el potencial de las actuaciones docentes para la mejora educativa en el área de Lenguaje y Comunicación (PAI-CONICYT n 82140013), financiado por el Concurso Nacional de Apoyo al Retorno de Investigadores desde el Extranjero, convocatoria 2014. Comisión Nacional de Investigación Científica y Tecnológica, Gobierno de Chile.

II- Universidad Austral de Chile, Valdivia Chile.Contacto: fmunita@gmail.com
}

\section{Resumen}

La formación de lectores conforma uno de los objetivos socioeducativos más relevantes que las sociedades contemporáneas le asignan a la escuela. En los últimos años, la investigación y la experiencia educativa han demostrado que una de las claves para avanzar en este propósito es la atención a la lectura literaria en tanto espacio privilegiado para el desarrollo de hábitos y competencias de lectura durante la escolaridad obligatoria. Hoy en día, contamos con un campo científico que se ocupa específicamente de la investigación en este dominio: la didáctica de la literatura. El presente artículo aborda el proceso de creación y progresiva consolidación de esta disciplina en el marco general de la investigación educativa. Para ello, y a partir de una amplia investigación bibliográfica, este ensayo sintetiza las principales fuentes de origen de la didáctica de la literatura, las dificultades que ha sorteado en su proceso de formación y, finalmente, ofrece un detallado panorama del modelo de enseñanza - aprendizaje de la literatura consensuado por la comunidad científica en nuestros días. El texto concluye subrayando la necesidad de atender a la información proveniente de las prácticas reales de educación literaria en las aulas, como factor central en la consolidación disciplinar de la didáctica de la literatura.

\section{Palabras clave}

Investigación educativa - Didáctica de la literatura Educación literaria. 


\title{
The teaching of literature: towards a consolidation of the field'
}

Felipe Munita"

\begin{abstract}
Undoubtedly, the formation of readers is one of the most relevant social educational objectives that contemporary societies assign to school. In recent years, research and educational experience have shown that one of the keys to progress in this purpose is the attention paid to literary reading, as a privileged opportunity for developing reading habits and skills during compulsory schooling. Nowadays, we have access to a scientific field that deals specifically with this research domain: the teaching of literature. This article addresses the creation and progressive consolidation of this discipline in the general framework of educational research. For this, and building on extensive literature research, this essay summarizes the main sources of the didactics of literature, and the difficulties that have been weathered in its formation. Finally, it offers a detailed overview of the model of teaching and learning of literature, agreed on by the scientific community today. The text concludes by emphasizing the need to address the information resulting from the "actual" practice of literary education in the classroom as a central factor in the consolidation of the teaching of literature as a discipline
\end{abstract}

\section{Keywords}

Educational research - Teaching literature - Literary education.

I- This article is part of the research project El efecto profesor en la formación de lectores: Un estudio exploratorio sobre el potencial de las actuaciones docentes para la mejora educativa en el área de Lenguaje y Comunicación (PAI-CONICYT n 82140013) (PAI-CONICYT $n^{\circ}$ 82140013), funded by Concurso Nacional de Apoyo al Retorno de Investigadores desde el Extranjero, 2014 call. Comisión Nacional de Investigación Científica y Tecnológica. Chilean government.

II- Universidad Austral de Chile, Valdivia, Chile. Contact: fmunita@gmail.com 
Introducción: espacios de formación de la disciplina

Los intentos por construir un aparato conceptual que explique los diversos fenómenos implicados en la enseñanza-aprendizaje de contenidos en las aulas, surgen en la década de 1970 en el ámbito de la didáctica de las ciencias y de las matemáticas. Son, podría decirse, el punto de partida de la didáctica en tanto disciplina científica, es decir, entendida no únicamente como un espacio de actuación sino también como un campo de investigación cuyo objeto es construir un cuerpo de conocimientos que ayuden a profundizar nuestra comprensión de la acción pedagógica y, de ese modo, hacerla avanzar hacia modelos educativos más afınados $\mathrm{y}$ fundamentados.

Más de 40 años después, el vasto corpus de investigación acumulado nos permite contar con un conjunto de saberes cada vez más amplio y coherente sobre su objeto de estudio. Nos permite, por ejemplo, consensuar una definición de las didácticas entendidas como "disciplinas de investigación que analizan los contenidos (saberes, saber hacer...) en tanto objetos de enseñanza-aprendizaje referidos/referibles a materias escolares" ${ }^{1}$ (REUTER, 2007, p. 69). Es decir, su especificidad radica precisamente en su interés por la relación entre contenidos y procesos de enseñanza-aprendizaje, desmarcándose así de otras disciplinas que estudian uno $\mathrm{u}$ otro de estos componentes, pero no la interacción entre ambos.

En ese nuevo contexto científico, durante las últimas dos décadas del siglo XX se desarrolló una didáctica específica de la lengua y la literatura, que si bien comparte el interés común por el sistema didáctico como objeto central de estudio, se diferencia de las didácticas de las ciencias a la hora de delimitar sus contenidos de enseñanza. Pues, en su caso, dichos contenidos no remiten únicamente a los saberes de los campos científicos de

1- Las traducciones de citas escritas originalmente en otras lenguas son nuestras. referencia (las ciencias del lenguaje y la teoría literaria, fundamentalmente), sino también a las prácticas relacionadas con los usos sociales de la lectura y escritura (CAMPS, 2000). Como bien lo explican Bronckart y Schneuwly:

[...] en materia de enseñanza de lenguas, particularmente de enseñanza de la lengua materna, los objetivos tienen relación con prácticas (orales o escritas) y con actitudes (normas), al menos tanto como con un saber propiamente dicho. En este terreno, la definición del contenido a enseñar opera, pues, a la vez a solicitud de los corpus científicos y a solicitud de las prácticas sociales de referencia. (1996, p. 71).

La complejidad de la interacción entre saberes científicos y prácticas sociales constituiría, entonces, una especificidad de la didáctica de la lengua y la literatura, disciplina que debe enfrentar el desafío de conjugar la adquisición de los saberes sabios con la apropiación de los quehaceres del lector (LERNER, 2001), entendiendo esto último como un complejo entramado de contenidos que incluye un saber hacer, unos valores y actitudes, así como unas formas de relación con y hacia la cultura escrita.

En suma, un primer conjunto de factores contextuales que inciden en la formación de la disciplina remite al proceso de construcción y progresiva consolidación del territorio científico de las didácticas, en general, y de la didáctica de la lengua, en particular, terrenos que conforman el marco global en el cual se desarrollan los nuevos enfoques para la enseñanza de la literatura. Ahora bien, si la didáctica de la lengua es, como hemos visto, un campo científico reciente, la didáctica de la literatura lo es todavía más, y el propio proceso de búsqueda de identidad de esta ha pasado por la problematización de su relación -más o menos autónoma- con aquella (DAUNAY, 2007). Así, si en los años 90 se hablaba de la necesidad de articular procesos de definición sobre la especificidad de una didáctica de la literatura (REUTER, 1995), a día de hoy se asume 
su entidad en tanto campo específico al interior de la didáctica de la lengua (DUFAYS, 2007), con su propio conjunto de saberes, preguntas de investigación, actores y redes de estudio. Sin embargo, para entender las lógicas propias del actual campo de la didáctica de la literatura no basta con atender al desarrollo científico de las didácticas, sino que es necesario observar otros factores que, vistos en una dimensión diacrónica, permiten una mayor comprensión de los procesos de evolución y desarrollo de esta disciplina.

Hay un amplio consenso (BOMBINI, 2008; COLOMER, 1996; 2005; DAUNAY, 2007) en torno a situar sus orígenes en la crítica formulada entre los años sesenta $\mathrm{y}$ setenta al modelo tradicional de enseñanza de la literatura en la escuela. Heredado de la tradición decimonónica, este modelo de tipo historicista se centraba en la transmisión del patrimonio literario entendido como puerta de acceso a la cultura, y encontraba en la lectura intensiva de clásicos y literaturas nacionales seguida de ejercicios explicativos por parte del profesorado- el mejor medio para lograr sus propósitos. Sin embargo, la sensación de fracaso de un modelo de lectura que ya no respondía a las nuevas realidades sociales (nuevo alumnado en las aulas y nuevas funciones delegadas a la escuela, al menos) favoreció la emergencia de otras perspectivas didácticas. La enseñanza se vio entonces centrada en el análisis detallado de los textos, para el cual se tomaron prestados los procedimientos provenientes de las corrientes formalistas y estructuralistas (DUFAYS; GEMENNE; LEDUR, 2005). De este modo entró a las escuelas el complejo mecanismo de análisis denominado comentario de texto, orientado a construir una lectura crítica y distanciada basada en la identificación de los recursos literarios utilizados por el autor. Una lectura, en suma, que al centrarse casi exclusivamente en un nivel metaliterario dejaba muy poco espacio para la actividad de construcción de sentido por parte del lector.

Paralelamente a estos cambios en las aulas, en la teoría de la literatura surgieron aportaciones que acabarian siendo fundamentales en los procesos de renovación didáctica. La atención hacia la actividad de lectura, particularmente a la relación de interacción y cooperación entre el lector y el texto en la construcción de significados, encuentra importantes formulaciones en la estética de la recepción (ISER, 1987) y la semiótica textual (ECO, 1981). A su vez, estas perspectivas teóricas ofrecían claras líneas de continuidad con otro espacio fundamental para la nueva didáctica: la teoría transaccional o de respuestas lectoras (ROSENBLATT, 2002), que concibe el acto de lectura como una situación orgánica afectada por las contribuciones del texto y del lector.

Así, los aportes de la teoría literaria hicieron que, desde los años ochenta, el acto de lectura y los procesos de comprensión e interpretación que el lector pone en juego pasaran a ser la principal preocupación de la didáctica (DUFAYS; GEMENNE; LEDUR, 2005). La ruptura epistemológica que significaba el desplazamiento del estudio del texto hacia el estudio del lector (ROUXEL, 1996), junto con la reconsideración (alentada desde la pragmática) de los diversos elementos del sistema literario en tanto factores que inciden en los procesos de lectura, conformaron finalmente las piedras angulares para un nuevo modelo de enseñanza de la literatura. En otras palabras, se construye así una didáctica que, más allá del texto, se orienta "tanto hacia la consideración de los factores externos del funcionamiento social del fenómeno literario, como hacia los factores internos de construcción del significado por parte del lector" (COLOMER, 2001, p. 8).

\section{Avances y dificultades en el proceso de transición de la disciplina}

Los procesos de evolución y desarrollo descritos más arriba quedaron, finalmente, estampados en una imagen asaz sugerente 
para explicar el cambio de paradigma: la del paso de una enseñanza de la literatura, centrada en la transmisión de conocimientos sobre el autor y el texto, a una educación literaria orientada a la formación de una competencia específica de lectura (COLOMER, 1991). Enunciada también como una formación para la recepción lectora (MENDOZA, 2002), esta nueva perspectiva tiene como propósito central ayudar a los estudiantes a progresar, durante toda la escolaridad obligatoria, en sus posibilidades de interpretación y de disfrute en tanto lectores literarios.

El interés por el lector y su actividad resulta, pues, evidente en tanto núcleo de estudio de la nueva didáctica. ¿Cuáles son, en ese marco, los principales problemas que ha enfrentado la educación literaria en el trayecto hacia un nuevo modelo de enseñanza? La respuesta comienza en el constato del importante vacío en la configuración de la disciplina escolar y de los saberes enseñables que trajo consigo la crítica al modelo historicista (BOMBINI, 2008). En poco tiempo, la didáctica de la literatura había quedado desprovista de los contenidos que tradicionalmente se consideraron fundadores de su existencia en tanto materia escolar, y fue necesario recurrir a nuevos modelos para suplir ese vacío.

Además de la emergencia de la vertiente estructuralista mencionada más arriba, una nueva perspectiva sumaba adeptos para reconfigurar el lugar de la literatura en la enseñanza. Se trata del denominado modelo comunicativo, de importante presencia escolar en los últimos lustros del siglo XX (y aún hoy, en muchos contextos), que orientaba el aprendizaje de la lectura hacia los textos funcionales $\mathrm{y}$ al dominio de los diversos géneros discursivos propios de la vida en sociedad. Y si bien este modelo implicaba grandes avances en relación al estudio de los textos en su función y usos sociales, el lugar de la literatura quedaba, no obstante, en un plano secundario, subsumido como uno más entre los géneros discursivos $\mathrm{y}$, por tanto, con un tratamiento didáctico similar al de otros textos como los informativos, argumentativos o prescriptivos. Esto llevó, finalmente, a trabajar el texto literario como un simple soporte de actividades lingüísticas (TAUVERON, 1999), situación que no considera un aprendizaje específico para favorecer la adquisición escolar de una competencia literaria.

Por otra parte, la emergencia de un nuevo contexto de promoción de la lectura y su progresiva incidencia en la programación escolar, hizo que la creación de hábitos lectores y la consecución del placer de la lectura entraran a formar parte de los nuevos objetivos sociales encargados a la escuela. Podría decirse que este movimiento surge como reacción natural hacia un modelo de enseñanza basado en el traspaso de conocimientos (COLOMER, 2001) y en la poca consideración del alumno en tanto sujeto lector. En ese marco debe entenderse el amplio llamado a desescolarizar la lectura que ha caracterizado el discurso de muchos mediadores desde el último cuarto del pasado siglo. Se entronizó así el acercamiento lúdico y libre a los textos por sobre la lectura obligatoria guiada. A su vez, esto derivó en una auténtica pedagogía del placer (BOMBINI, 2001) muy influenciada por los postulados de Pennac (1992) y sus derechos del lector, presentada como una forma de animación a la lectura que respondería mejor al objetivo social de formar hábitos lectores durante la etapa escolar. Y si bien este movimiento incidió positivamente afirmando la importancia del acceso directo de los alumnos a los textos $\mathrm{y}$ creando espacios de intercambio entre lectores en las aulas, trajo también efectos negativos sobre los procesos de aprendizaje que debían asociarse a esas instancias. Pues, al basar la lectura literaria en el contacto libre con los textos, la intervención docente pasó a considerarse un obstáculo en la relación placentera del niño con el libro, lo que trajo consigo una manera liviana de leer los textos literarios (BAJOUR, 2009) que vivió su auge 
en los años noventa y se extendió hasta los primeros lustros del nuevo siglo.

El debate originado a partir de estas experiencias de animación discurría, entonces, sobre la pregunta acerca de si la lectura literaria es enseñable o únicamente opera por contagio. Evidentemente, la adhesión a la idea del contagio implicaba un lugar secundario para las actividades específicas de aprendizaje de la lectura literaria, tal como sucedía (aunque por distintas razones) en el enfoque comunicativo. Así, la expansión de la idea del contagio supuso cierto retraso en la consolidación de una educación literaria entendida como proceso de aprendizaje, mediado por el docente, y orientado al desarrollo de una competencia interpretativa que permitiera a los alumnos leer obras progresivamente más complejas en lugar de reducir el placer a un restringido corpus de textos fáciles.

En ese contexto, la idea de que los libros no se trabajan, se disfrutan, adquirió fuerza y pasó a conformar una de las grandes tensiones que hasta el día de hoy dificultan la consolidación del nuevo modelo de educación literaria. Pues, si en la actualidad hay consenso en torno a la importancia del placer de leer como aspecto central en la lectura literaria, también lo hay en relación a la imposibilidad de fundar una didáctica únicamente con el placer como finalidad. Entre las numerosas limitaciones que esto ofrecería, rescatamos aquí dos de las desarrolladas por Dufays, Gemenne y Ledur (2005) que nos parecen especialmente relevantes. La primera: sabemos que el placer no depende únicamente de factores escolares, sino que está fuertemente condicionado por elementos socioculturales; ergo, una didáctica fundada únicamente en las posibilidades de fruición literaria que cada alumno trae consigo favorecería a aquellos alumnos (los herederos) que en su entorno han tenido múltiples posibilidades de encuentro $\mathrm{y}$ de socialización con los textos, y haría muy difícil la experimentación de ese placer en quienes provengan de entornos alejados de la práctica lectora. Segundo: el mencionado placer suele asociarse exclusivamente a un solo tipo de fruición, aquella nacida de la identificación y la participación emocional en el texto; por tanto, su formulación como objetivo educativo no permitiría avanzar hacia otras formas más distanciadas de placer que también hacen parte de la experiencia literaria.

En síntesis, el vacío de saberes escolares en el que se encontró la didáctica de la literatura tomó diversas direcciones cuyos enfoques se han hecho presentes de un modo $\mathrm{u}$ otro (y hasta el día de hoy) en nuestras escuelas. Por una parte, la atención hacia la lectura funcional en el modelo comunicativo; por otra, el interés de los nuevos mediadores por favorecer hábitos de lectura placentera en las aulas. A ello debemos sumar el fuerte arraigo que el enfoque estructuralista encontró en la enseñanza secundaria, situación que impuso el comentario de textos como principal dispositivo de la nueva didáctica, y el dominio del aparato conceptual asociado a la literatura como su principal objetivo. Tres orientaciones cuya asunción ofreció importantes avances en relación al modelo tradicional, pero que implicaban también nuevos problemas $\mathrm{y}$ tensiones para la formación de lectores literarios en la escuela.

Con todo, la visión panorámica recién esbozada nos lleva a pensar que la actual didáctica de la literatura parece fundarse sobre dos grandes críticas, posteriores a aquella formulada al modelo historicista. La primera es la crítica referida al saber conceptual sobre literatura como fin en sí mismo o como objeto exclusivo de la didáctica. Podría sintetizarse en la idea de que "en la escuela no se aprende de qué hablan las obras, sino de qué hablan los críticos" (TODOROV, 2007, p. 22), constato cuya necesidad de superación hoy resulta evidente. La segunda es la crítica referida al placer en tanto objetivo que, como vimos, no basta para articular una didáctica tendiente a formar lectores literarios que diversifiquen sus posibilidades de interpretación y fruición 
de los textos. Podría sintetizarse en la idea de que los objetivos educativos consisten en ayudar al niño a construir las herramientas de sus placeres, y no a la obligación de experimentarlos (COLOMER, 2001), y en la conciencia de que la lectura literaria implica un placer "estético, intelectual y cultural que, lejos de operar por magia, se construye" (TAUVERON, 2002, p. 14).

\section{El nuevo modelo de educación literaria}

Los nuevos objetivos adscritos a la educación literaria parecen orientados hacia dos grandes aspectos (COLOMER, 2001): por una parte, se trataría de favorecer el avance en la competencia interpretativa; por otra, promover la construcción de hábitos lectores así como una relación placentera y de implicación personal frente a los textos. Lo anterior, que implica la superación tanto de una enseñanza literaria basada únicamente en el saber como de otra basada solo en el placer, permite avanzar en la concreción de objetivos que atiendan a las nuevas demandas sociales, y favorece un proceso formativo tendiente a lograr que los alumnos participen con efectividad en los procesos de recepción del discurso literario.

Una segunda formulación que, en cierto modo, aglutina los dos componentes de la primera, la encontramos en la idea de que enseñar literatura es, en principio, enseñar "una manera de leer" (DUFAYS; GEMENNE; LEDUR, 2005, p. 123). Es decir: no es el dominio de un patrimonio de obras, ni la apropiación de los valores morales que estas vehiculan, ni el análisis de las características formales de su literariedad el objetivo último de la nueva didáctica, sino la progresiva adquisición de un modo de leer por parte de los alumnos, un saber leer literario que, al finalizar su escolaridad, puedan poner en juego en sus lecturas libres y personales.

La cuestión, entonces, es definir y caracterizar esa postura de lectura que estaría en el centro del actual paradigma de educación literaria. Ayuda, en primer lugar, pensarla en relación a la construcción escolar de una competencia literaria, entendida como el "conjunto de saberes que permiten leer e interpretar un texto literario" (MENDOZA, 2004, p. 71) y como la "capacidad de acceder a la lectura de obras cada vez más complejas o a lecturas cada vez más complejas de las obras" (JOVER, 2010, p. 29). No obstante, para una comprensión más completa de lo que esa postura pone en juego debemos recuperar otro concepto clave del nuevo modelo: el de lectura literaria. Tal como señala Daunay (2007) esta noción se ha construido como reemplazo y superación de la idea de literariedad, permitiendo concebir un nuevo objeto de enseñanza que, lejos de basarse en los elementos que conformarían el carácter literario de una obra, se centra ahora en la relación establecida entre el lector y el texto.

En esa línea, Dufays, Gemenne y Ledur (2005) han defendido una concepción de la lectura literaria entendida como un va y viene dialéctico entre participación y distanciación, es decir, una aproximación que incluye tanto la apropiación subjetiva, espontánea y emocional propia de la participación psicoafectiva en el texto, como una recepción de orden intelectual y distanciada, basada en procedimientos racionales de análisis e interpretación de las obras. Así, la dialéctica entre los aspectos pasionales $\mathrm{y}$ racionales del juego literario emerge como la modalidad de lectura que encarna los nuevos objetivos educativos. En palabras de Tauveron (2002, p. 321) "es la conjugación de las dos posturas, fusional y analítica, lo que permite una real apropiación del texto". Pues, si de lo que se trata es de formar lectores literarios que se sientan implicados en la lectura, y que además puedan poner en juego formas variadas de comprensión y fruición sobre los textos, trabajar en la adquisición y el progresivo dominio de esta forma de lectura parece ser el mejor aporte que pueda hacer la escuela en la formación lectora de las nuevas generaciones. 
Uno de los principales consensos del discurso didáctico contemporáneo (COLOMER, 2005; JOOLE, 2006; TAUVERON, 2002) radica en pensar la educación literaria desde la continuidad de objetivos entre los diversos niveles de escolaridad. Esto implica asumir los propósitos anteriormente enunciados como orientaciones que sirven tanto para favorecer los primeros acercamientos al discurso literario en educación infantil como para construir los dispositivos didácticos de lectura para jóvenes que terminan su enseñanza obligatoria. Evidentemente, lo anterior echa por tierra la extendida creencia en torno a que se aprende a disfrutar en primaria y a leer de forma distanciada y analítica en secundaria, y deja en el centro del quehacer docente el desarrollo progresivo de una competencia literaria a construir durante toda la etapa escolar.

Por tanto, resulta fundamental la reflexión en torno a esas líneas de continuidad que asegurarían la formación escolar del lector literario. Una de las aportaciones más destacadas en este sentido es la de Colomer (2005), con su planteamiento de siete líneas de progreso en el aprendizaje escolar de la lectura literaria. En esta propuesta, dicho aprendizaje pasaría por favorecer el avance de los estudiantes en dominios tan diversos como el grado de implicación personal con la literatura, el desarrollo de habilidades de interpretación inferencial de las obras, o la diversificación de las formas de fruición propias del lector literario.

Sin duda, lo anterior ha ayudado en el proceso de concreción de los principales desafíos que enfrenta la educación literaria. No obstante, ha sido necesario concretar aún más la programación considerando los objetivos específicos que guían la actuación educativa. En ese marco, es importante recordar que, si bien los objetivos de desarrollo de la competencia interpretativa y de construcción del hábito lector son complementarios y deben planificarse en conjunto, ambos necesitan de estrategias diferenciadas y comportan diferentes modalidades de lectura: una lectura analítica y guiada, en el primer caso, y una lectura autónoma, en el segundo. A su vez, ambas modalidades están relacionadas con una determinada intencionalidad: "la voluntad de analizar y de estudiar los textos en profundidad, por una parte, o bien la de trasladar a la escuela los rituales de las prácticas privadas y sociales de lectura, por otra" (MANRESA, 2013, p. 62). Asimismo, cada una de estas dimensiones tiene su propio espacio de desarrollo: mientras la lectura por placer y la construcción del hábito encuentran en la biblioteca (escolar o de aula) su medioambiente más natural, la competencia interpretativa se adquiere fundamentalmente en el contexto de la clase de lengua y literatura (MARGALLO, 2012).

La cuestión, entonces, es indagar en los ejes programáticos atendiendo ahora a la especificidad de cada uno de estos propósitos. Es así como, a partir de diversas aportaciones (MANRESA, 2013; MARGALLO, 2012; TAUVERON, 1999; 2002), hemos construido un cuadro comparativo (tabla 1) que favorece la visualización de la especificidad de cada objetivo, atendiendo a criterios tan diversos como el corpus literario, los espacios y dispositivos didácticos, la postura de lectura privilegiada y la actividad mediadora: 
Tabla 1- Cuadro comparativo objetivos de la educación literaria.

\begin{tabular}{|c|c|c|}
\hline & DESARROLLO DE LA COMPETENCIA INTERPRETATIVA & FORMACIÓN DEL HÁBITO LECTOR \\
\hline 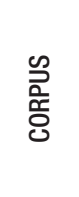 & $\begin{array}{l}\text { Ofrecer textos resistentes desde un inicio, es decir, textos que } \\
\text { presentan ciertos problemas de comprensión o que invitan } \\
\text { al lector a comprometerse en la resolución de problemas de } \\
\text { interpretación. Un corpus que favorece el esfuerzo intelectual y } \\
\text { una postura activa de interrogación del texto como bases de la } \\
\text { construcción de sentido. }\end{array}$ & $\begin{array}{l}\text { La palabra clave es diversidad; se trata de ofrecer un menú } \\
\text { lo más variado posible de lecturas (integrando, también, } \\
\text { las lecturas aportadas por los propios alumnos, y teniendo } \\
\text { siempre en cuenta sus perfiles en tanto lectores), para } \\
\text { llevarlos a experiencias placenteras de lectura. }\end{array}$ \\
\hline 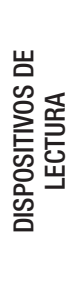 & $\begin{array}{l}\text { Se basa en espacios de lectura obligatoria guiada, que permiten } \\
\text { el estudio colectivo y en profundidad de los textos. Esto } \\
\text { implica construir dispositivos didácticos tendientes a activar el } \\
\text { proceso interpretativo (tanto en el espacio individual como en la } \\
\text { socialización de significado entre lectores) y hacerlo avanzar hacia } \\
\text { interpretaciones progresivamente más complejas y ajustadas a } \\
\text { las claves del texto. A su vez, los dispositivos siempre toman en } \\
\text { cuenta las características, problemas y posibilidades que cada } \\
\text { texto ofrece para el desarrollo de la competencia literaria. }\end{array}$ & $\begin{array}{c}\text { Habilitar tiempos de lectura individual y silenciosa, así como } \\
\text { otros de lectura en voz alta por parte del docente y otros para } \\
\text { compartir y socializar las lecturas personales. Dinamizar los } \\
\text { espacios de lectura mediante dispositivos genéricos que se } \\
\text { quieren similares a las prácticas sociales de lectura, como la } \\
\text { recomendación de textos entre pares o la descripción de los } \\
\text { libros a partir de consignas sencillas que otorguen amplios } \\
\text { márgenes para hablar sobre ellos. }\end{array}$ \\
\hline 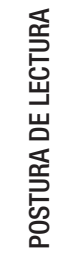 & $\begin{array}{l}\text { Se trata de activar y construir un protocolo singular de lectura, } \\
\text { basado en un progresivo equilibrio entre los derechos del texto (el } \\
\text { horizonte interpretativo construido por la obra) y los derechos del } \\
\text { lector (la activa participación que se le exige en la construcción } \\
\text { de significado). Esto implica reconocer que, si bien todas las } \\
\text { interpretaciones son posibles, siempre hay unas más aceptables } \\
\text { que otras al momento de apoyarlas en los elementos textuales y } \\
\text { las claves de lectura ofrecidas por las obras. }\end{array}$ & $\begin{array}{c}\text { Se trata de recuperar la postura lectora más corriente del } \\
\text { ámbito extraescolar: la respuesta emocional y afectiva hacia } \\
\text { los textos, para darle lugar en el horario escolar e intentar } \\
\text { enriquecerla educando la capacidad de disfrutar las lecturas } \\
\text { desde diversas formas de aproximación y apropiación del } \\
\text { texto. }\end{array}$ \\
\hline 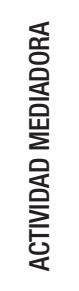 & $\begin{array}{c}\text { El docente planifica espacios que permitan la emergencia, } \\
\text { circulación y confrontación de las interpretaciones de los alumnos, } \\
\text { y favorece la reflexión metacognitiva sobre el trabajo interpretativo } \\
\text { allí construido, con la finalidad de que aprendan a evaluar la } \\
\text { pertinencia de sus interpretaciones y a hacerlas dialogar con las } \\
\text { de otros. Administra las instancias de discusión sobre las obras } \\
\text { actuando como garante de los derechos del texto, y favorece } \\
\text { espacios de sistematización y adquisición de los saberes literarios } \\
\text { puestos en juego. }\end{array}$ & $\begin{array}{l}\text { El docente es quien debe garantizar y facilitar el acceso a } \\
\text { la amplia diversidad de textos que este objetivo educativo } \\
\text { requiere. En ese marco, algunas de sus principales funciones } \\
\text { serían: potenciar un ambiente cultural en el cual se lee, se } \\
\text { comparten las lecturas y se favorece la participación en } \\
\text { verdaderos circuitos sociales de circulación de la literatura y } \\
\text { las producciones culturales; reforzar la autoimagen lectora } \\
\text { de sus alumnos; y ayudarles en la elección de los textos } \\
\text { atendiendo a sus necesidades y habilidades de lectura. }\end{array}$ \\
\hline
\end{tabular}

Fuente: Cuadro elaborado por el autor.

Hacia la concreción de un modelo didáctico y de sus dispositivos de referencia

Si, como hemos visto, la didáctica de la lengua y la literatura remite a la vez a saberes científicos y a prácticas sociales de lectura y escritura, la concreción de nuevos modelos didácticos ha intentado atender a esa especificidad poniendo en el centro del proceso educativo unas tareas de producciónrecepción textual con verdaderos propósitos comunicativos para el alumno. De este modo, se lee y se escribe en el aula de manera similar a como se lee y escribe fuera de ella, es decir, con una intencionalidad determinada, enmarcada en una situación comunicativa que le da sentido al esfuerzo que estas prácticas exigen.

Así, el nuevo paradigma de educación literaria encontró en el trabajo por proyectos su mejor y más natural línea de desarrollo. Desde esta perspectiva, la actuación educativa se basa en la formulación e implementación de secuencias didácticas (CAMPS, 1994), entendidas como una propuesta de creación de un producto textual concreto, cuya recepción se dará en el contexto de una situación comunicativa real y cuya elaboración precisa el aprendizaje de determinados conocimientos literarios. De este modo, la adquisición y dominio de los saberes disciplinares quedan al servicio de una experiencia comunicativa, 
y el proceso de enseñanza-aprendizaje deviene espacio de múltiples actividades de lectura y escritura necesarias para la consecución de los propósitos comunicativos consensuados al interior del grupo.

Con todo, lo que las secuencias didácticas brindan a la enseñanza literaria es un marco global cuya realización se apoya en diversas modalidades de lectura y en el uso de dispositivos didácticos que encarnen las orientaciones esbozadas más arriba. En palabras de Tauveron (2002), la educación literaria necesita dispositivos fértiles, es decir, que inviten a leer, que permitan identificar y resolver problemas de comprensión, y que favorezcan procesos de interacción tanto del alumno con el texto como de los alumnos entre sí en torno a la lectura.

En ese marco, los procesos de renovación de los dispositivos de referencia de la didáctica de la literatura han derivado en la creación y progresiva consolidación de nuevos géneros disciplinares (DIAS-CHIARUTTINI, 2015), entre los cuales la discusión literaria ${ }^{2}$ ocupa un lugar especialmente destacado.

En términos generales, cabría emparentar la discusión con un tipo de habla exploratoria que toma lugar en las aulas. Esto es, un diálogo cooperativo basado en la confrontación de diversos puntos de vista, en el respeto y consideración de la opinión del otro, y en la progresiva construcción de acuerdos y consensos en el marco de un razonamiento colectivo (MERCER; LITTLETON, 2007). Ya en el ámbito de la educación literaria, entendemos la discusión como un espacio de construcción de sentido en el seno de un círculo de lectura (CHAMBERS, 2007; TERWAGNE; VANHULLE; LAFONTAINE, 2003) definido por el intercambio y la interacción con otros (los pares, el profesor y el texto) y cuyo propósito es poner en juego múltiples puntos de vista sobre una lectura. Se trata, pues, de una situación dialógica, de coconstrucción acumulativa de los sentidos de un

2- Conocida como débat interprétatif en el ámbito francófono, y literary discussion en el anglosajón. texto, gestionada por el mediador y orientada a hacer emerger la comprensión de niñas y niños a partir de la confrontación de las diversas interpretaciones surgidas en un grupo ante una lectura compartida.

Así, la movilización de ideas, la pluralidad de miradas y la negociación de sentido parecen ser sus características más destacadas, las que podríamos profundizar atendiendo a dos aspectos clave. Primero, el lugar central del texto, que adopta una doble función de puerto de partida y de brújula durante el trayecto interpretativo pues, finalmente, son los índices textuales los que ayudan a justificar las respuestas. Segundo, la concepción positiva de las proposiciones que contradicen el texto, vistas no ya como errores a subrayar o perseguir, sino como aportaciones que forman parte del hilo evolutivo de una discusión. Aportaciones que, en un proceso de gestión del error interpretativo por parte del docente (DIASCHIARUTTINI, 2015), serán sometidas a la confrontación con los otros y con el texto para su posterior validación o rechazo.

La gestión de la discusión es, precisamente, uno de los grandes desafíos de un dispositivo que nace desde un cierto vacío en la tradición pedagógica, que nunca se ocupó de articular conversaciones literarias guiadas y orientadas hacia la adquisición de determinados saberes conceptuales, procedimentales y actitudinales como los que pone en juego la lectura compartida. En este punto, una de las propuestas de mayor interés ha sido la de Chambers (2007), quien plantea la puesta en escena de diversos tipos de preguntas sobre el libro, desde unas más genéricas como lo que ha gustado o lo que ha desconcertado, hasta otras más específicas que apuntan a trabajar tanto sobre las particularidades de cada texto como sobre la respuesta personal que los niños han elaborado en su lectura.

La discusión literaria parece, pues, el género disciplinar de referencia de la nueva didáctica de la literatura. Esto se complementa con otros dispositivos que han demostrado su interés en la investigación y experimentación 
reciente, entre los cuales destacamos el carnet lector, la autobiografía lectora, los escritos de trabajo y los fórums literarios.

Señalado como el complemento ideal para la discusión literaria (TERWAGNE; VANHULLE; LAFONTAINE, 2003), el carnet lector podría definirse como "un instrumento escolar orientado, no al aprendizaje de saberes declarativos, sino a la formación de sujetos lectores capaces de considerar con reflexividad su lectura empírica del texto" (AHR, 2011, p. 161). Hablamos aquí de un diario de lectura que, al estar centrado en la recepción empírica y en la apropiación personal de las obras, le otorga una entidad didáctica a las primeras impresiones, sensaciones, divagaciones y pensamientos poco estructurados que caracterizan toda actividad de recepción lectora.

A su vez, su maridaje con procesos de discusión literaria permite al alumno reconsiderar su lectura personal a la luz de la construcción colectiva de significado, y elaborar así nuevas aproximaciones más distanciadas y orientadas al análisis crítico. En ese sentido destaca la noción de carnet de semillas (TERWAGNE; VANHULLE; LAFONTAINE, 2003), que concibe el dispositivo como un repositorio de primeras impresiones que se desea compartir con el grupo, reflexiones que se espera crezcan $y$ fructifiquen en el debate. Así, copias de extractos o frases que han sido especialmente relevantes para el alumno, sus opiniones o reflexiones personales sobre el texto o las ideas que les ha evocado un pasaje determinado serían algunas de las posibles entradas para el carnet o diario de lectura.

El constato acerca de que la imagen de sí en tanto lector es un importante factor en la motivación e implicación por la lectura (BAYE; LAFONTAINE; VANHULLE, 2003) llevó también a pensar dispositivos que focalizaran especialmente en ese punto. Esto derivó, entre otras líneas de avance, en la escolarización de la autobiografía lectora, que en tanto género disciplinar didáctico ofrece un doble potencial (MUNITA, 2013). Por una parte, le permite al profesor saber dónde se está: conocer los bagajes de lectura del grupo es un primer paso para articular los itinerarios y las actividades de interpretación con las experiencias lectoras que trae el alumnado, y con sus posibilidades de valoración y apreciación literaria. $\mathrm{Al}$ alumno, por su parte, le ofrece una instancia de reflexión y toma de conciencia a partir de la evocación de sus itinerarios de lectura, instancia que en muchas ocasiones deviene espacio de producción de una autoimagen lectora que, en palabras de Rouxel, es "un acto fundador de la identidad del lector” (2004, p. 47).

Si bien no tienen una entidad de género disciplinar como los otros aquí evocados, los diversos escritos de trabajo puestos en juego durante la lectura de textos literarios representan dispositivos didácticos especialmente útiles como medios para construir la comprensión e interpretación. Son concebidos como escritos "transitorios y efímeros, al servicio de la elaboración del pensamiento y del intercambio de opiniones" (TAUVERON, 2002, p. 167), y pueden adoptar múltiples formas, desde preguntas formuladas por los alumnos hacia el propio texto o esquemas para afinar una interpretación inicial hasta escritos para situar al estudiante en el rol del escritor o, incluso, para permitirle expresar su visión sobre los objetivos de lectura explicitados por el docente.

Finalmente, el cruce entre la consabida importancia de la socialización en la formación del lector y el actual contexto socioeducativo de expansión de las tecnologías de la información y la comunicación ha devenido un escenario especialmente interesante para la didáctica de la literatura. Los nuevos contextos comunicativos que la red ofrece permiten abrir espacios de diálogo e intercambio sobre las obras, concretados fundamentalmente en la participación y creación de fórums literarios (MANRESA, 2013) orientados a la movilización tanto de lecturas autónomas como escolares, y a la proyección de las opiniones y reacciones personales sobre esas obras. A su vez, estos nuevos espacios cuentan con un importante 
valor agregado para la didáctica: el carácter público de la escritura, aspecto que mediatiza la opinión personal y que, eventualmente, podría favorecer instancias de trabajo sobre las producciones de los alumnos.

Visto este panorama, podría decirse que la investigación ha realizado importantes avances dirigidos a poner en escena unos instrumentos mediadores coherentes con los nuevos objetivos formulados. Todos ellos, en las diversas variantes que puedan presentar, son potencialmente útiles para los dos grandes objetivos formativos de la educación literaria. No obstante, hay algunos que por sus características son especialmente interesantes para uno u otro objetivo. A modo de ejemplo: mientras la discusión literaria y los escritos de trabajo presentan un enfoque mayoritario (aunque no exclusivo) hacia el desarrollo de la competencia interpretativa, la autobiografía lectora o los fórums en la red apuntan, en cambio, a la construcción de hábitos lectores y a la consolidación de las prácticas sociales y autónomas de lectura.

\section{A modo de conclusión}

El itinerario seguido en estas páginas ha permitido visualizar las principales líneas de avance de la didáctica de la literatura como un campo disciplinar de relevancia en el amplio contexto de la investigación educativa, y que responde a uno de los objetivos prioritarios que las sociedades contemporáneas le asignan a la escuela: la formación de lectores.

Ahora bien, pese al espacio de consolidación disciplinar aquí reseñado, la didáctica de la literatura debe hacer frente a realidades educativas que todavía parecen muy lejanas de los consensos aportados desde la investigación. Sabemos, por ejemplo, que la formulación del nuevo modelo de enseñanza ha tenido escasa repercusión en los currícula escolares, pues aunque los documentos oficiales de diversos países han incorporado entre sus objetivos la necesidad de formar lectores con hábitos de lectura consolidados y capacidad crítica para entender los textos literarios, muchos de sus programas no llegan a concretar las vías que facilitarian el desarrollo de esos objetivos (DURÁN; MANRESA, 2009). Por su parte, las investigaciones sobre las prácticas efectivas y sus resultados de aprendizaje alertan una y otra vez sobre el extendido fracaso en la formación de lectores durante la escolaridad obligatoria (BAYE; LAFONTAINE; VANHULLE, 2003; MANRESA, 2013).

En esa línea, nos parece que el camino más atractivo para transitar en los próximos años, lejos de alimentar el extendido discurso alarmista sobre los escasos hábitos lectores de la población escolar, es el de generar estudios tendientes a observar y analizar lo que está en juego en las prácticas reales de enseñanza de la literatura en las aulas. Es decir, indagar en las múltiples formas de concreción que la educación literaria adopta cuando se la observa en situación, para, de este modo, avanzar hacia una comprensión más ajustada y profunda sobre los diversos factores que inciden en la formación escolar del lector literario. Para ello, parece especialmente interesante analizar los resultados reportados por aquellas investigaciones que, como los estudios de caso o la investigación - acción, ofrecen miradas particulares sobre estos procesos, focalizadas en experiencias de aulas concretas de las cuales se pueden extraer múltiples aprendizajes.

Afortunadamente, este tipo de estudios es cada vez más frecuente en nuestro campo. Sería, pues, esperable que en los próximos años la confrontación de resultados de investigaciones desarrolladas en esa línea ayudara a enriquecer el enfoque actual de la educación literaria que, como todo modelo de enseñanza - aprendizaje, es perfectible y está sujeto a continuos procesos de mejora. 


\section{Referencias}

AHR, Sylviane. Le carnet de lecteur de littérature au collège et au lycée: un dispositif expérimental misant sur une nouvelle approche de l'interaction lecture-écriture. In: AEBY DAGHÉ, Sandrine (Dir.). Enseigner la langue et la littérature: des dispositifs pour penser leur articulation. Namur: Presses Universitaires de Namur, 2011. p. 157-175.

BAJOUR, Cecilia. ¿Qué tiene que ver la promoción de la lectura con la escuela? Imaginaria, Buenos Aires, n. 259, 2009. Disponible en: <http://www.imaginaria.com.ar/2009/11/\%C2\%BFque-tiene-que-ver-la-promocion-de-la-lectura-con-laescuela/>. Acceso en: 22 jun. 2015.

BAYE, Ariane; LAFONTAINE, Dominique; VANHULLE, Sabine. Lire ou ne pas lire: état de la question. Les Cahiers du C.L.P.C.F. 4. Bruxelles: Centre de Lecture Publique de la Communauté Française, 2003.

BOMBINI, Gustavo. La literatura en la escuela. In: ALVARADO, Maite (Coord.). Entre líneas: teorías y enfoques en la enseñanza de la escritura, la gramática y la literatura. Buenos Aires: Flacso; Manantial, 2001. p. 53-74.

BOMBINI, Gustavo. Volver al futuro: postales de la enseñanza literaria. In: LOMAS, C (Ccoord.). Textos literarios y contextos escolares. Barcelona: Graó, 2008. p. 135-147.

BRONCKART, Jean Paul; SCHNEUWLY, Bernard. La didáctica de la lengua materna: el nacimiento de una utopía indispensable. Textos de Didáctica de la Lengua y la Literatura, Barcelona, n. 9, p. 61-78, 1996.

CAMPS, Anna. Introducció: objecte, modalitat i àmbits de la recerca en didàctica de la llengua. In: CAMPS, Anna; RíOS, Isabel; CAMBRA, Margarida (Coord.). Recerca y formació en didàctica de la llengua. Barcelona: Graó, 2000. p. 9-22.

CAMPS, Anna. Projectes de llengua entre la teoria i la pràctica. Articles de Didàctica de la llengua i la literatura, Barcelona, n. 2, p. 7-20, 1994.

CHAMBERS, Aidan. Dime: los niños, la lectura y la conversación. México, DF: Fondo de Cultura Económica, 2007.

COLOMER, Teresa. Andar entre libros: la lectura literaria en la escuela. México, DF: Fondo de Cultura Económica, 2005.

COLOMER, Teresa. De la enseñanza de la literatura a la educación literaria. Comunicación, Lenguaje y Educación, Madrid, n. 9, p. 21-31, 1991.

COLOMER, Teresa. La didáctica de la literatura: temas y líneas de investigación e innovación. In: LOMAS, Carlos (Coord.). La educación lingüística y literaria en la educación secundaria. Barcelona: ICE-UAB; Horsori, 1996. p. 123-142.

COLOMER, Teresa. La enseñanza de la literatura como construcción de sentido. Lectura y Vida, Buenos Aires, v. 22, n. 1, p. 6-23, 2001.

DAUNAY, Bernard. Le sujet lecteur: une question pour la didactique du français. Le Français Aujourd'hui, Paris, n. 157, p. 43-51, 2007

DIAS-CHIARUTTINI, Ana. Le débat interprétatif dans l'enseignement du français. Berne: Peter Lang, 2015.

DUFAYS, Jean-Louis. Du sens à l'évaluation, en passant par l'utilité: actualité et enjeux d'une problematique de recherche. In: DUFAYS, Jean-Louis (Ed.). Enseigner et apprendre la littérature aujourd'hui, pour quoi faire? Louvain-la-Neuve: Presses Universitaires de Louvain, 2007. p. 7-15.

DUFAYS, Jean-Louis; GEMENNE, Louis; LEDUR, Dominique. Pour une lecture littéraire: histoire, théories, pistes pour la classe. Bruxelles: De Boeck, 2005.

DURÁN, Carme; MANRESA, Mireia. Entre países: la acción educativa en nuestro entorno. In: COLOMER, Teresa (Coord.). Lecturas adolescentes. Barcelona: Graó, 2009. p. 59-116.

ECO, Umberto. Lector in fabula: la cooperación interpretativa en el texto narrativo. Barcelona: Lumen, 1981. 
ISER, Wolfgang. El acto de leer: teoría del efecto estético. Madrid: Taurus, 1987.

JOOLE, Patrick. Lire des récits longs. Paris: Retz-CRDP Versailles, 2006.

JOVER, Guadalupe. La educación literaria en el bachillerato: futuros posibles. Textos de Didáctica de la Lengua y la Literatura, Barcelona, n. 55, p. 27-38, 2010.

LERNER, Delia. Leer y escribir en la escuela: Io real, lo posible y lo necesario. México, DF: Fondo de Cultura Económica, 2001.

MANRESA, Mireia. L'univers lector adolescent: dels hàbits de lectura a la intervenció educativa. Barcelona: Rosa Sensat, 2013.

MARGALLO, Ana Maria. Claves para formar lectores adolescentes con talento. Leer.es, Madrid, 2012. Disponible en: <http://www. educando.edu.do/files/1013/3433/5687/Claves_para_formar_lectores.pdf>. Acceso en: 22 jun. 2015.

MENDOZA, Antonio. La educación literaria: bases para la competencia lecto-literaria. Málaga: Aljibe, 2004.

MENDOZA, Antonio. Las funciones del profesor de Literatura: bases para la innovación. In: MUÑOZ, Belén et al. Aspectos didácticos de lengua y literatura/ 12. Zaragoza: ICE Universidad de Zaragoza, 2002. p. 109-140.

MERCER, Neil; LITTLETON, Karen. Dialogue and the development of children's thinking. New York: Routledge, 2007.

MUNITA, Felipe. Jo, lector: els relats de vida lectora en la construcció del subjecte didàctic. Articles de Didàctica de la llengua i la literatura, Barcelona, n. 61, p. 17-25, 2013.

PENNAC, Daniel. Comme un roman. Paris: Gallimard, 1992.

REUTER, Yves (Ed.). Dictionnaire des concepts fondamentaux des didactiques. Bruxelles: De Boeck, 2007.

REUTER, Yves. Pour une didactique de la littérature: la nécessaire définition des biens littéraires. In: POSLANIEC, Christian (Ed.). Littérature et jeunesse. Paris: INRP, 1995. p. 129-145.

ROSENBLATT, Louise. La literatura como exploración. México, DF: Fondo de Cultura Económica, 2002.

ROUXEL, Annie. Enseigner la lecture littéraire. Rennes: Presses Universitaires de Rennes, 1996.

ROUXEL, Annie. Lecture et retour sur soi: l'autobiographie de lecteur au lycée. Enjeux, Namur, n. 61, p. 47-65, 2004.

TAUVERON, Caterine. Comprendre et interpréter le littéraire à l'école: du texte réticent au texte proliférant. Repères, Lyon, n.19, p. 9-38, 1999.

TAUVERON, Caterine (Coord.). Lire la littérature à l'école. Paris: Hatier, 2002.

TERWAGNE, Serge; VANHULLE, Sabine; LAFONTAINE, Annette. Les cercles de lecture: interagir pour développer ensemble des compétences de lecteurs. Bruxelles: De Boeck, 2003.

TODOROV, Tzevtan. La literatura en peligro. Barcelona: Galaxia Gutenberg; Círculo de lectores, 2007.

Recibido en: 07.07.2015

Aprobado en:16.02.2016

Felipe Munita es doctor en Didáctica de la lengua y la literatura por la Universidad Autónoma de Barcelona (UAB), con maestrías en Literatura infantil y juvenil y Promoción de la lectura. Profesor de Castellano y licenciado en Letras (PUC-Chile). Miembro del grupo GRETEL de la Universidad Autónoma de Barcelona. Actualmente es investigador asociado en el Instituto de Lingüística y Literatura de la Universidad Austral de Chile. 